EPJ manuscript No.

(will be inserted by the editor)

\title{
Josephson oscillation of a superfluid Fermi gas
}

\author{
Sadhan K. Adhikaria \\ Instituto de Física Teórica, UNESP - São Paulo State University, 01.405-900 São Paulo, São Paulo, Brazil
}

November 13, 2018

\begin{abstract}
Using the complete numerical solution of a time-dependent three-dimensional mean-field model we study the Josephson oscillation of a superfluid Fermi gas (SFG) at zero temperature formed in a combined axially-symmetric harmonic plus one-dimensional periodic optical-lattice (OL) potentials after displacing the harmonic trap along the axial OL axis. We study the dependence of Josephson frequency on the strength of the OL potential. The Josephson frequency decreases with increasing strength as found in the experiment of Cataliotti et al. [Science 293 (2001) 843] for a Bose-Einstein condensate and of the experiment of Pezzè et al. [Phys. Rev. Lett. 93 (2004) 120401] for an ideal Fermi gas. We demonstrate a breakdown of Josephson oscillation in the SFG for a large displacement of the harmonic trap. These features of Josephson oscillation of a SFG can be tested experimentally.
\end{abstract}

PACS. 03.75.Ss Degenerate Fermi gases, 03.75.Lm Tunneling, Josephson effect, Bose-Einstein condensates in periodic potentials, solitons, vortices, and topological excitations, 03.75.Kk Dynamic properties of condensates; collective and hydrodynamic excitations, superfluid flow

\section{Introduction}

The observation of an oscillating Josephson current across an one-dimensional (1D) periodic array of potential wells, usually generated by a polarized standing-wave laser field and commonly known as an optical-lattice (OL) potential, in a trapped "cigar-shaped" Bose-Einstein condensate (BEC) by Cataliotti et al. [1] was the first manifestation of this phenomenon in trapped neutral bosons. Until then the Josephson effect was confirmed in superconductors with charged electrons and in liquid helium [1,2]. In the experiment of Cataliotti et al. [1] a Josephson oscillation was initiated in a repulsive ${ }^{87} \mathrm{Rb}$ BEC formed in a 1D periodic OL plus an axially-symmetric harmonic potentials by suddenly displacing the harmonic trap along the axial direction. They found that the Josephson frequency reduced with the increase of the strength of the OL potential. Also a breakdown of Josephson oscillation was observed for large displacement of the OL potential.

There have been theoretical studies of Josephson oscillation using the numerical solution of the time-dependent mean-field Gross-Pitaevskii (GP) equation 3] in one (1D) [1,4] and three (3D) [5,6] dimensions to understand different experimental features [1. There have also been other theoretical studies of Josephson oscillation in a trapped BEC [7.8] using different approaches and under different conditions distinct from the experiment of Cataliotti et al. [1]. Moreover, there have been many interesting studies on

a Electronic address: adhikari@ift.unesp.br; URL: http://www.ift.unesp.br/users/adhikari/ different aspects of Josephson oscillation in a cold Fermi gas under diverse conditions of trapping [9].

Recently, in another experiment on ideal Fermi gas of ${ }^{40} \mathrm{~K}$ atoms in an axially-symmetric plus OL trap Pezzè et al. [10] observed the oscillation of the Fermi gas after a sudden displacement of its equilibrium position along the lattice for various OL strength. They measured the frequency of oscillation for various OL strength. They also explained the result of experiment using a semiclassical theory.

Due to a strong repulsive Pauli-blocking interaction at low energies among spin-polarized fermions, there cannot be an evaporative cooling leading to a quantum degenerate Fermi gas (DFG) [1]. Trapped DFG has been achieved only by sympathetic cooling in the presence of a second boson or fermion component. Recently, there have been successful observation [11,12,13,14 and associated experimental 15, 16, 17, and theoretical [20, 21, 22, 18, 19, studies of BEC-DFG mixtures. Different experimental groups 11, 12, 13, 14] observed the formation of a DFG in the following systems: ${ }^{6,7} \mathrm{Li}$ [13], ${ }^{23} \mathrm{Na}-{ }^{6} \mathrm{Li}$ [14] and ${ }^{87} \mathrm{Rb}-{ }^{40} \mathrm{~K}[15$, 16. Also, there have been studies of a degenerate mixture of two hyperfine-spin components of fermionic ${ }^{40} \mathrm{~K}[11$ and ${ }^{6} \mathrm{Li} 12$ atoms. Later on, the observation 23 of the transition of a DFG to a Bardeen-Cooper-Schrieffer (BCS) superfluid fermion gas (SFG) of opposite hyperfine-spin orientation by manipulating the fermion-fermion interaction using a Feshbach resonance has opened the possibility of controlled study of a BCS superfluid formed under the action of a weak fermion-fermion attraction. The use of a Feshbach resonance has also allowed the observation 
S. K. Adhikari: Josephson oscillation of a superfluid Fermi gas

of BCS-BOSE crossover 24 in two-hyperfine-component fermion vapors of ${ }^{40} \mathrm{~K}\left[25,23\right.$, and ${ }^{6} \mathrm{Li}[26$ atoms.

There have been theoretical models of a DFG using a mean-field hydrodynamic approch based essentially on the Thomas-Fermi-Weizsäcker approximation 27, 18. Such models have provided results 19 for collapse in a boson-fermion mixture of ${ }^{87} \mathrm{Rb}^{-}{ }^{40} \mathrm{~K}$ in qualitative agreement with the observation by Modugno et al. 15, 22. There have also been studies of fermionic bright [28], dark [29], and gap solitons 30 in a boson-fermion mixture where the bosonic component is treated by the mean-field GP equation [3] and the fermionic component by a hydrodynamic model [19, 18 . (The Feshbach resonances in the boson-fermion systems ${ }^{23} \mathrm{Na}-{ }^{6} \mathrm{Li}$ and ${ }^{87} \mathrm{Rb}^{40} \mathrm{~K}$ have been observed experimentally 31 and can be used in a controlled experiment of solitons in such a mixture.) The results of such studies are in agreement with $a b$ initio studies of gap 32 and bright 33 solitons based on properly antisymmetrized many-body approach.

Although a mean-field-hydrodynamical approach to a DFG has its limitations (as in the absence of a coherent phase it may determine only the fermion density), a Ginzburg-Landau-type mean-field description 34 of a BCS superfluid based on a Lagrangian density is theoretically well founded and widely appreciated and has been used 30,35] in dealing with superfluid boson-fermion mixtures (such a description for a SFG determines the fermion density as well as the phase of a complex coherent order parameter). A rigorous antisymmetrized many-body approach for a SFG becomes unfeasible as the number of fermions increases, where the simplified mean-field approach of a BCS superfluid in terms of a one-body wave function of Cooper-paired fermions is of advantage.

In this paper we study the Josephson oscillation of a BCS superfluid at zero temperature in an OL potential using the complete numerical solution of a 3D mean-field model. As in the experiment on Josephson oscillation of a BEC [15], we consider a SFG formed in a cigar-shaped axially-symmetric parabolic trap with an added OL potential along the axial direction. The Josephson oscillation is initiated by giving a translation of the parabolic trap in the axial direction upon the formation of the SFG. The SFG acquires energy in the process to initiate the Josephson oscillation. We study the variation of the frequency of Josephson oscillation for different OL strength. We also study the breakdown of smooth oscillation for large initial displacement of the parabolic trap. We compare our results for frequency with experimental results on superfluid Bose ${ }^{87} \mathrm{Rb}$ atoms [1] as well as on Fermi ${ }^{40} \mathrm{~K}$ atoms [10.

The present model is derived as the Euler-Lagrange equation of a Lagrangian density using the well-known energy density of a SFG [36. The Euler-Lagrange equation so obtained is a nonlinear partial differential equation with a nonlinearity of power $7 / 3$. Such an equation has also been used for a DFG with a different coefficient multiplying the nonlinearity (the coefficient in the two cases is different due to the Cooper pairing in a BCS superfluid).
In Sec. II we present the mean-field model for a SFG mixture. In Sec. III we present numerical results for Josephson oscillation of a SFG in a combined harmonic plus 1D OL potentials as a function of the strength of the OL when the harmonic trap is displaced through a small distance along the optical axis. We also illustrate the breakdown of Josephson oscillation in a SFG when the oscillation is initiated by giving a large displacement of the harmonic trap along the optical axis. Finally, in Sec. IV we present a summary of our study.

\section{Mean-field Model for Fermi superfluid}

Let us consider a BCS superfluid Fermi gas (SFG) of $N$ Cooper-paired fermions of mass $m$. The energy density of the system is given by $\mathcal{E}=3 \sigma \epsilon_{F} / 5$ [36], where $\epsilon_{F} \equiv$ $\hbar^{2} k_{F}^{2} /(2 m)$ is the Fermi energy with $k_{F}$ the Fermi momentum and $\sigma$ the atomic density. (Here we neglect small corrections to this expression due to the residual fermionfermion interaction usually expressed as an expansion in scattering length.) Modification to this expression appropriate to study a BCS-Bose crossover 24] has also been suggested [37. The atomic density for the superfluid corresponding to the isotropic distribution (spherical Fermi surface) is $\sigma=2(2 \pi)^{-3} \int_{0}^{k_{F}} 4 \pi k^{2} d k \equiv\left(3 \pi^{2}\right)^{-1}\left(2 m \varepsilon_{F} / \hbar^{2}\right)^{3 / 2}$, where the effect of Cooper pairing is included in the extra factor of 2. This relation can be inverted to yield $\epsilon_{F}=\hbar^{2}\left(3 \pi^{2} \sigma\right)^{2 / 3} /(2 m)$, which leads to the following expression for energy density of the system

$$
\mathcal{E}=\frac{3 \hbar^{2}\left(3 \pi^{2}\right)^{2 / 3}}{10 m} \sigma^{5 / 3} .
$$

In the spirit of the Ginzburg-Landau theory 34 the SFG can be described by a complex order parameter $\Psi$, such that $\sigma=\Psi^{2}$. In terms of this order parameter, the Lagrangian density of the superfluid can be written as

$$
\begin{aligned}
\mathcal{L} & =\frac{i \hbar}{2}\left(\Psi^{*} \frac{\partial \Psi}{\partial t}-\Psi \frac{\partial \Psi^{*}}{\partial t}\right)-\frac{\hbar^{2}}{2 m_{\mathrm{eff}}}|\nabla \Psi|^{2}-V(\mathbf{r}) \Psi \\
& -\frac{3 \hbar^{2}\left(3 \pi^{2}\right)^{2 / 3}}{10 m} \Psi^{10 / 3},
\end{aligned}
$$

where $V(\mathbf{r})$ is the external potential, and $m_{\text {eff }}$ is the effective mass of superfluid flow as in the Ginzburg-Landau theory. (The exact value of $m_{\mathrm{eff}}$ is not known but there is evidence [34 that for a Cooper-paired SFG it is twice the fermion mass and we shall use this value in the following: $\left.m_{\mathrm{eff}}=2 m\right)$. The Euler-Lagrange equation of Lagrangian density (2) becomes the following 3D nonlinear Schrödinger equation with a repulsive nonlinear term of power $7 / 3$ :

$$
i \hbar \Psi_{t}=\frac{-\hbar^{2}}{2 m_{\mathrm{eff}}} \nabla^{2} \Psi+\frac{\hbar^{2}}{2 m}\left(3 \pi^{2}\right)^{2 / 3}|\Psi|^{4 / 3} \Psi+V(\mathbf{r}) \Psi,
$$

with normalization $\int|\Psi(\mathbf{r}, t)|^{2} d \mathbf{r}=N$. In the presence of the combined axially-symmetric and periodic OL potentials [1,5] $V(\mathbf{r})=\frac{1}{2} m_{\mathrm{eff}} \omega^{2}\left(\rho^{2}+\nu^{2} z^{2}\right)+V_{\mathrm{opt}}$, where 
$\omega$ is the angular frequency of the harmonic potential in the radial direction $\rho, \nu \omega$ that in the axial direction $z$, with $\nu$ the aspect ratio, and $V_{\text {opt }}=s E_{R} \cos ^{2}\left(k_{L} z\right)$ is the OL potential created with the standing-wave laser field of wavelength $\lambda$, with $E_{R}=\hbar^{2} k_{L}^{2} /(2 m), k_{L}=2 \pi / \lambda$, and $s$ $(<12)$ the strength. Although, for small $s$ one should have a Joshepson oscillation, for large $s$ values Joshepson oscillation should terminate, as in the case of a BEC [38, due to a superfluid to Mott-insulator transition. (This transition cannot be studied with the mean-field equations and field-theoretic analysis is needed for its understanding.)

In the axially-symmetric configuration, in the zero angular momentum state the fermion order parameter can be written as $\Psi(\mathbf{r}, t)=\psi(\rho, z, t)$, where $0 \leq \rho<\infty$ and $-\infty<z<\infty$. Now transforming to dimensionless variables $\hat{\rho}=\sqrt{2} \rho / l, \hat{z}=\sqrt{2} z / l, \tau=t \omega, l \equiv \sqrt{\hbar /\left(m_{\mathrm{eff}} \omega\right)}$, and $\varphi(\hat{\rho}, \hat{z} ; \tau) \equiv \hat{\rho} \sqrt{l^{3} /(N \sqrt{8})} \psi(\rho, z ; t)$, Eq. (3) becomes 6. 19

$$
\begin{aligned}
& {\left[-i \frac{\partial}{\partial \tau}-\frac{\partial^{2}}{\partial \hat{\rho}^{2}}+\frac{1}{\hat{\rho}} \frac{\partial}{\partial \hat{\rho}}-\frac{\partial^{2}}{\partial \hat{z}^{2}}+\frac{1}{4}\left(\hat{\rho}^{2}+\nu^{2} \hat{z}^{2}\right)-\frac{1}{\hat{\rho}^{2}}\right.} \\
& \left.+\frac{V_{\mathrm{opt}}}{\hbar \omega}+n\left|\frac{\varphi(\hat{\rho}, \hat{z} ; \tau)}{\hat{\rho}}\right|^{4 / 3}\right] \varphi(\hat{\rho}, \hat{z} ; \tau)=0
\end{aligned}
$$

where the nonlinearity parameter $n=m_{\mathrm{eff}}\left(3 \pi^{2} N\right)^{2 / 3} / m$. In terms of the 1D probability $P(z, t) \equiv 2 \pi \int_{0}^{\infty} d \hat{\rho}|\varphi(\hat{\rho}, \hat{z}, \tau)|^{2} F \dot{p}$, $r$ t we consider a SFG formed in the combined harmonic the normalization of the wave function is given by $\int_{-\infty}^{\infty} d \hat{z} P(z$ and periodic OL potentials for a specific nonlinearity $n$. 1 .

In many of the experiments with DFG [11,15, 16], ${ }^{40} \mathrm{~K}$ fermion atoms were used and in this study we also consider ${ }^{40} \mathrm{~K}$ atoms and take $m$ to be the mass of $\mathrm{K}$ atoms. As in the experiment of Cataliotti et al. 1 the axial and radial trap frequencies are taken as $\nu \omega=2 \pi \times 9 \mathrm{~Hz}$ and $\omega=2 \pi \times 92 \mathrm{~Hz}$, respectively, with $\nu=9 / 92 \approx 0.1$. For ${ }^{40} \mathrm{~K}$, the harmonic-oscillator length $l=\sqrt{\hbar /\left(m_{\mathrm{eff}} \omega\right)} \approx 1$ $\mu \mathrm{m}$ and the present dimensionless length unit corresponds to $l / \sqrt{2} \approx 0.7 \mu \mathrm{m}$. The present dimensionless time unit corresponds to $\omega^{-1}=1 /(2 \pi \times 92) \mathrm{s}=1.73 \mathrm{~ms}$. Although we perform the calculation in dimensionless units using Eq. (4), we present the results in actual physical units using these conversion factors consistent with ${ }^{40} \mathrm{~K}$ atoms. We take the wavelength $\lambda$ of the standing-wave laser beam to make the OL potential to be $\lambda=700 \mathrm{~nm}$, so that the dimensionless laser wave length $\lambda_{0}=\sqrt{2} \lambda / l \simeq 1$ and the dimensionless standing-wave energy parameter $E_{R} /(\hbar \omega)=4 \pi^{2} / \lambda_{0}^{2}$. Hence in dimensionless units the OL potential of Eq. (4) is

$$
\frac{V_{\text {opt }}}{\hbar \omega} \equiv s \frac{E_{R}}{\hbar \omega} \cos ^{2}\left(k_{L} z\right)=s \frac{4 \pi^{2}}{\lambda_{0}^{2}}\left[\cos ^{2}\left(\frac{2 \pi}{\lambda_{0}} \hat{z}\right)\right] .
$$

Though most of our calculation was done with above set of parameters, for a comparison with the experiment on Fermi ${ }^{40} \mathrm{~K}$ atoms of Pezzè et al. 10, we also repeated our calculation with trap frequencies $\nu \omega=2 \pi \times 24 \mathrm{~Hz}$ and $\omega=2 \pi \times 275 \mathrm{~Hz}$, and OL wavelength $\lambda=863 \mathrm{~nm}$.

We solve the three-dimensional Eq. (4) numerically using a split-step time-iteration method with the CrankNicholson discretization scheme described recently 39.
We discretize the GP equation typically with time step 0.001 and space step 0.1 spanning $\rho$ from 0 to $7 \mu \mathrm{m}$ and $z$ from $-120 \mu \mathrm{m}$ to $120 \mu \mathrm{m}$, although, sometimes we used smaller steps for obtaining convergence. Equation (4) is then solved by time iteration starting with the known harmonic oscillator solution for $n=0: \varphi(\hat{\rho}, \hat{z})=\left[\nu /\left(8 \pi^{3}\right)\right]^{1 / 4}$ $\hat{\rho} e^{-\left(\hat{\rho}^{2}+\nu \hat{z}^{2}\right) / 4}$ with chemical potential $\mu=(1+\nu / 2)$ [40. For a typical cigar-shaped geometry with $\nu \simeq 0.1$ [1], $\mu(\simeq 1)$ is much smaller than the typical depth of the OL potential wells $E_{R} /(\hbar \omega)=4 \pi^{2} / \lambda_{0}^{2} \simeq 40$ so that $\mu<<E_{R} /(\hbar \omega)$ and the passage of fermion atoms from one well to other can only proceed through quantum tunneling [5,6]. During the time iteration of Eq. (44) the nonlinearity $n$ as well as the OL potential parameter $s$ are slowly increased by equal amounts in 100000 steps of time iteration until the desired value of nonlinearity and OL potentials are attained. Then, without changing any parameter, the solution so obtained is iterated 50000 times so that a stable solution is obtained independent of the initial input and time and space steps. The solution then corresponds to the bound SFG under the joint action of the harmonic and OL potentials.

\section{Numerical Results} monic and OL potentials of Eq. (5) for a range of values of $s$. In Fig. 1 (a) the plot of $|\psi(\rho, z)|$ vs. $\rho$ and $z$ illustrates the profile of the SFG for $N=40, n=225$ and $s=4$. From Fig. 1 (a) we see that the SFG has the shape of a cigar (of length $80 \mu \mathrm{m}$ and transverse radius $3 \mu \mathrm{m}$ ) cut into narrow slices with the OL barriers separating the slices. The large number of maxima and minima due to the OL potential is not clearly visible in this plot. The maxima and minima in the axial direction are clear in the plot of $|\psi(\rho, z)|$ vs. $\rho$ and $z$ in Fig. 1 (b) where we show the central part of function $|\psi(\rho, z)|$ for $3 \mu \mathrm{m}>z>-3 \mu \mathrm{m}$. In this interval of $z$, there are about 16 wells of the OL potential and as many maxima and minima in $|\psi(\rho, z)|$.

Now we consider an oscillating SFG in the combined harmonic and periodic OL potentials. If we suddenly displace the harmonic trap along the lattice axis by a small distance after the formation of the SFG in the combined potentials, the SFG will acquire a potential energy, be out of equilibrium and start to oscillate. As the height of the potential-well barriers of the OL potential is much larger than the chemical potential of the system, the atoms in the SFG will move by tunneling through the potential barriers. This fluctuating transfer of atoms across the potential barriers is due to Josephson effect in a neutral quantum SFG.

With the SFG of Fig. 1 (a) we next study its Josephson oscillation when the harmonic potential is suddenly displaced along the axial direction by $14 \mu \mathrm{m}$. The SFG now acquires an added potential energy which it can use to execute a Josephson oscillation along the axial direction. The Josephson oscillation is best studied numerically 

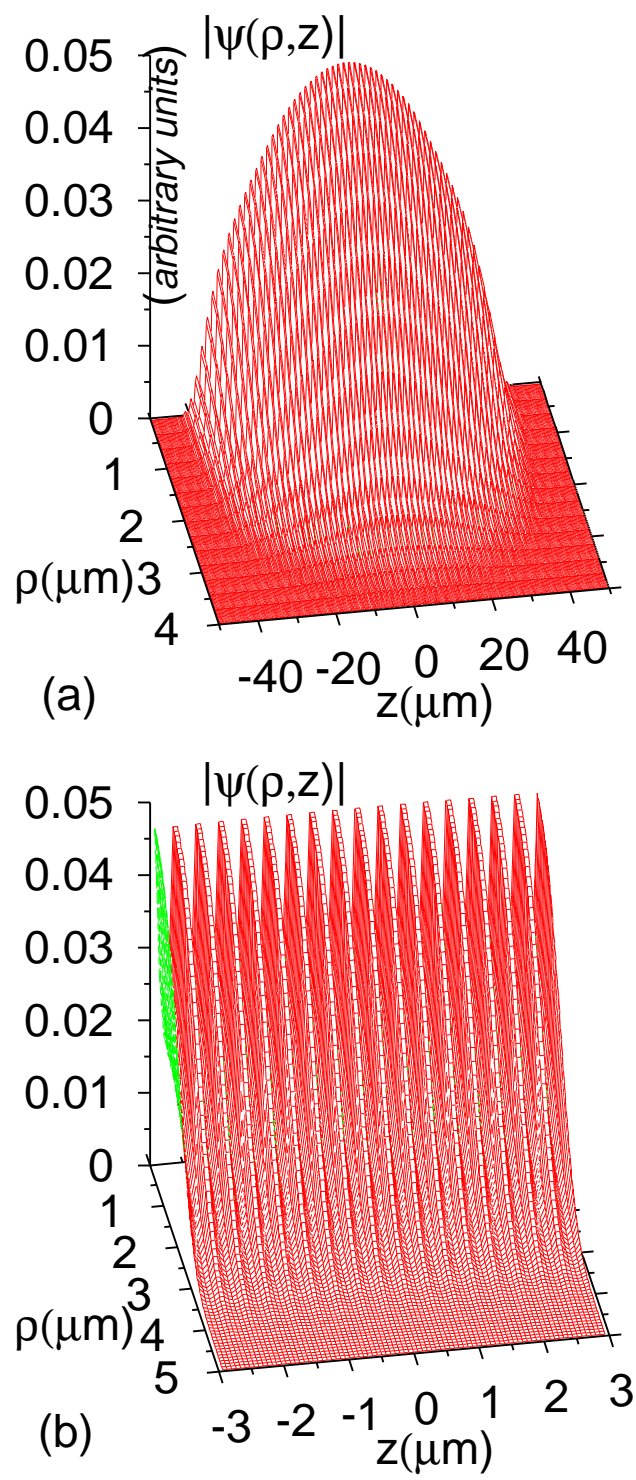

Fig. 1. (a) The profile of the SFG function $|\psi(\rho, z)|$ vs. $\rho$ and $z$ formed in the joint optical-lattice and harmonic potentials for $N=40, n=225$ and $s=4$. (b) The profile of the SFG function $|\psi(\rho, z)|$ of (a) vs. $\rho$ and $z$ for $3 \mu \mathrm{m}>z>-3 \mu \mathrm{m}$, which clearly exhibits the maxima and minima of the SFG function along the axial direction.

from the contour plot of the $1 \mathrm{D}$ probability $P(z, t)$ vs $z$ and $t$ exhibited in Figs. 2. These plots clearly show the central position of the SFG along the axial $z$ direction. In the present simulation we take different values of the OL strength $s$. These contour plots are very useful to find the Josephson frequencies. From Figs. 2 the periods of Josephson oscillation are easily read off and the frequencies calculated for different OL strengths $s$.

In Fig. 3 (a) we plot the Josephson frequencies vs. OL strength $s$. Specifically, in addition to the present calculation for SFG we also show (i) the experimental frequencies of Cataliotti et al. [1] for a repulsive BEC of ${ }^{87} \mathrm{Rb}$ atoms,
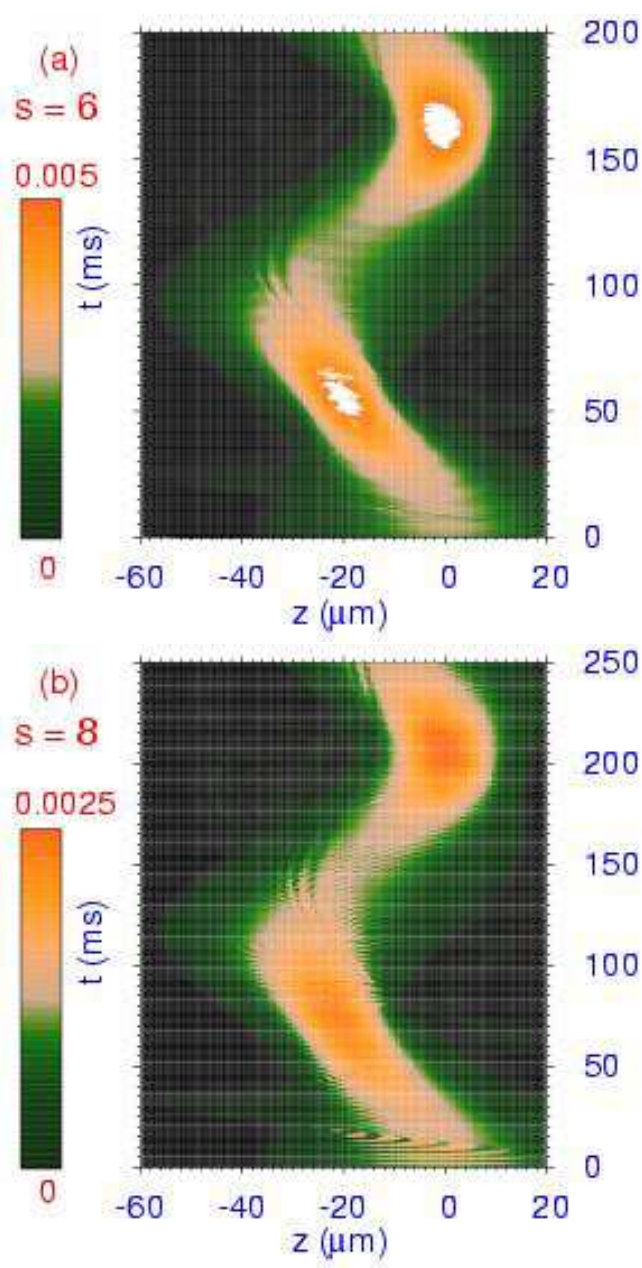

Fig. 2. (Color online) The contour plot of the $1 \mathrm{D}$ probability density $P(z, t)$ of the SFG with $n=225$ executing a Josephson oscillation when the harmonic trap is suddenly displaced along the axial direction through a distance $14 \mu \mathrm{m}$ at $t=0$ for OL strength $s=$ (a) 6 and (b) 8. The period of Josephson oscillation can be obtained from these plots.

(ii) the 3D simulation for a BEC from Ref. 6], and (ii) the hydrodynamical calculation for a BEC from Ref. [7. In Fig. 3 (b) we show the Josephson frequencies vs. OL strength with the parameters of the experiment of Pezzè et al. [10] for an ideal Fermi gas of ${ }^{40} \mathrm{~K}$ atoms. In this figure we compare our results with experiment and a semiclassical theoretical calculation 10.

The most interesting conclusion from Figs. 3 is that the present frequencies of 3D simulation for Josephson oscillation of a SFG are practically the same as the frequencies for Josephson oscillation of a BEC and close to those for oscillation of an ideal Fermi gas. For $s=0$ the OL potential is absent and the BEC and the SFG execute free oscillation with the frequency of the axial potential. From Figs. 3 we find that the Josephson frequency reduces with increasing OL strength. As the Josephson oscillation takes place by quantum tunneling of the atoms through the OL barriers, this oscillation is bound to be reduced as 

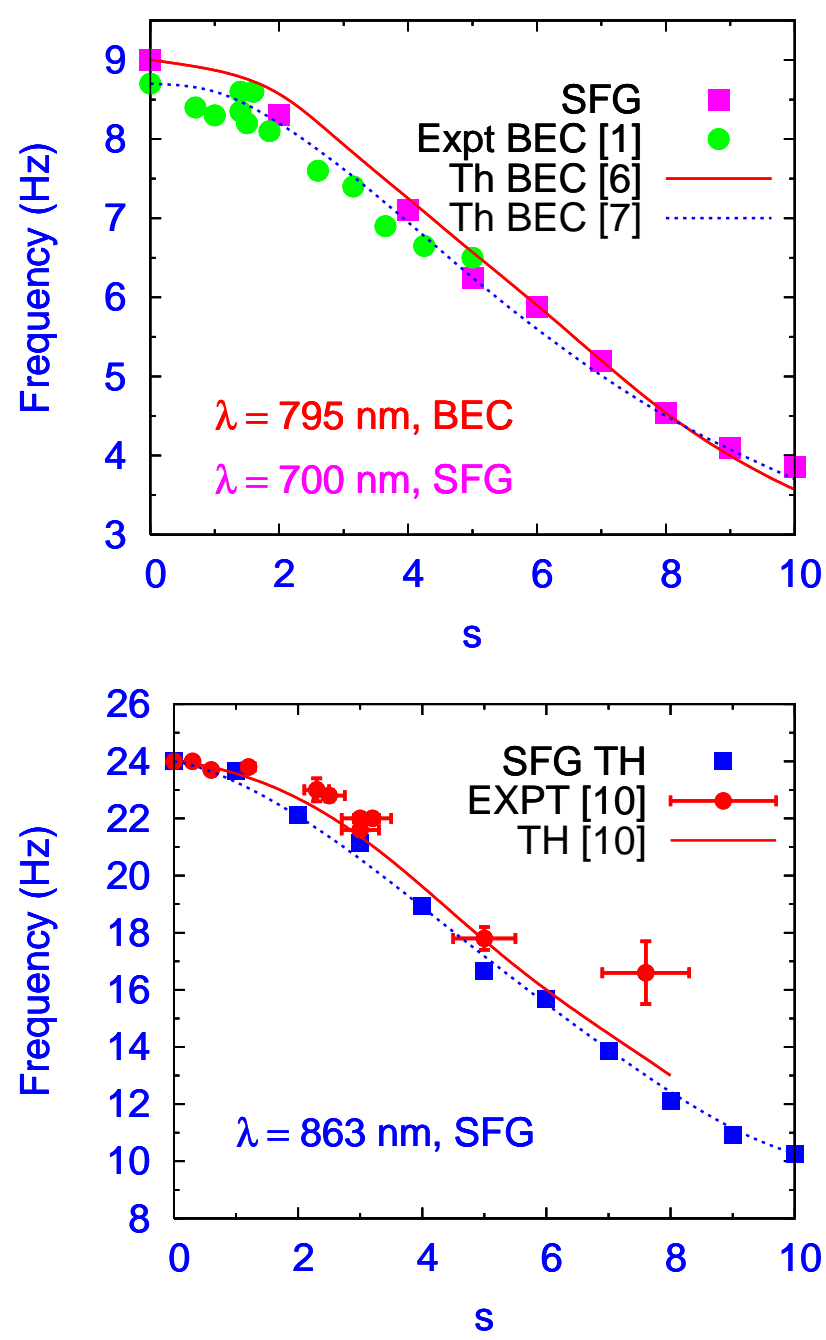

Fig. 3. (Color online) The frequency of the atomic current in the array of Josephson junctions as a function of OL strength $s$ compared with (a) experiment on boson ${ }^{87} \mathrm{Rb}$ and other theories (b) on fermion ${ }^{40} \mathrm{~K}$ and other theory. In (a) solid square - present result for SFG; solid circle - experiment of Cataliotti et al. 11 for BEC with repulsive ${ }^{87} \mathrm{Rb}$ atoms; full line - mean-field simulation for BEC [6]; dotted line - hydrodynamical calculation for BEC 7]. In (b) solid square - present result for SFG; solid circle - experiment of Pezzè et al. [10] for fermions with ${ }^{40} \mathrm{~K}$ atoms; full line - semiclassical theory of Pezzè et al. [10] for fermions; dotted line - joins present points to guide eye.

the height of the OL barriers is increased. The reduction of Josephson oscillation with the increase of the parameter $s$ results in a reduction of Josephson frequency in Fig. 3. The agreement of the frequencies of present model for superfluid Fermi gas with the experiment on ideal (nonsuperfluid) Fermi gas is remarkable. A future experiment on a superfluid Fermi gas may reveal subtle differences in behavior, if any, of such oscillations in superfluid and nonsuperfluid Fermi gas.
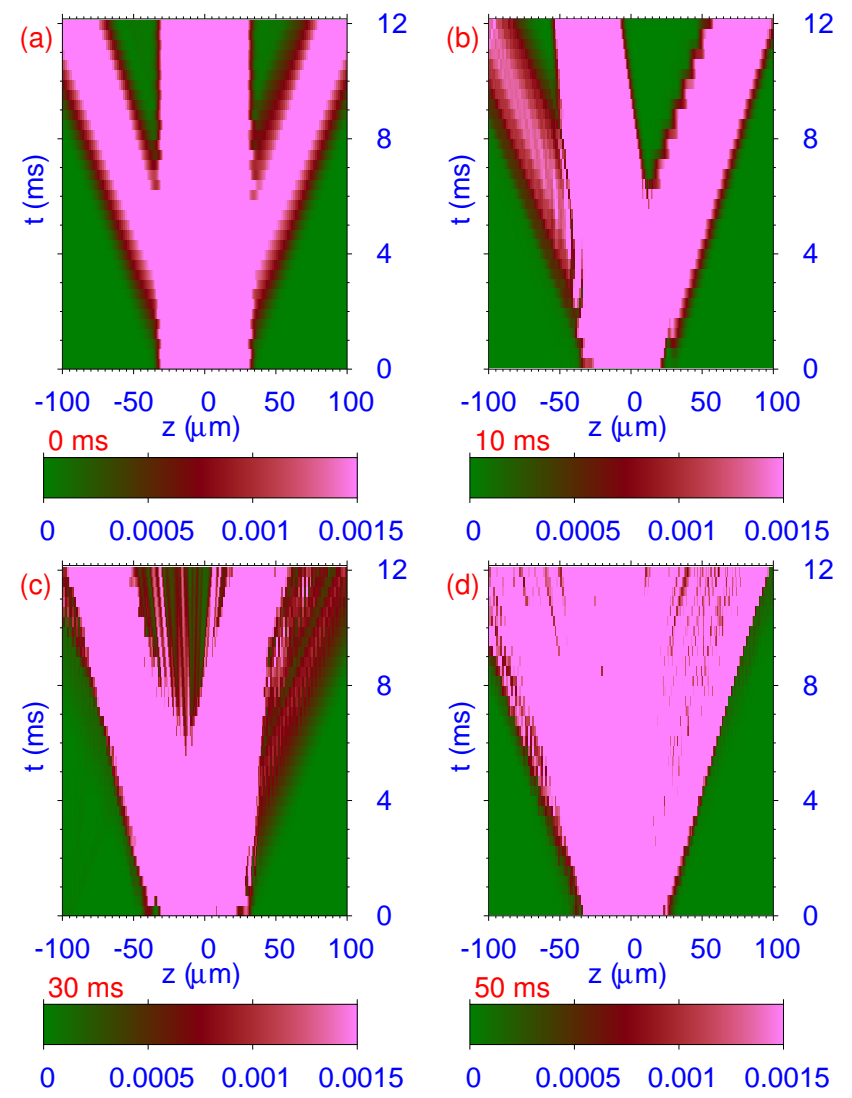

Fig. 4. (Color online) The contour plot of the 1D probability density $P(z, t)$ of the SFG with $n=225$ released after hold times $T_{h}=$ (a) $0 \mathrm{~ms}$, (b) $10 \mathrm{~ms}$, (c) $30 \mathrm{~ms}$, and (d) $50 \mathrm{~ms}$ when the harmonic trap is suddenly displaced along the axial direction through a distance $100 \mu \mathrm{m}$ for OL strength $s=8$. The three clean interference trails in (a) demonstrates the phase correlation in the initial SFG. With the increase of hold times in the displaced trap the clean interference trail is slowly lost in (b) and (c) signalling a loss in phase correlation. The phase correlation in the SFG is completely lost in (d) after a hold time of $50 \mathrm{~ms}$.

The SFG formed on the combined OL and harmonic traps considered so far in Figs. 1 and 2 is a phase-correlated quantum fluid with the atoms freely moving by quantum tunneling from one OL site to another at zero temperature and one has a phase-coherent macroscopic state. This will lead to an interference pattern when the SFG is released from the OL trap. However, the details of the pattern may change from one experiment to another. When the harmonic trap is displaced through a small distance as in Figs. 2, the SFG executes Josephson oscillation by quantum tunneling.

Next we consider an account of the breakdown of Josephson oscillation in a SFG when the harmonic potential is displaced by a very large distance $(\sim 100 \mu \mathrm{m})$ along the periodic OL potential. In that case for small times $(t<300$ ms) no Josephson oscillation of the type observed in Figs. 2 was found. The SFG was found to remain virtually fixed at $z=0$ for small times. At very large times it moved 
very slowly to the new center of equilibrium. However, the phase correlation of the SFG is lost after staying a significant time in the displaced trap. To demonstrate the destruction of phase correlation in the displaced trap we rely on the disappearance of the interference pattern formed upon the release of the SFG from the combined harmonic and OL traps after a hold time of $T_{h}$ in the displaced trap.

The formation of a BEC on a combined harmonic plus OL potentials and the interference pattern formed upon the release of this BEC from the confining traps have been studied both theoretically 4,42,41 and experimentally 1, 38, 43. We present a similar study for the SFG. The periodic OL potential cuts the SFG into several pieces at different sites maintaining phase correlation among them. As a result when the SFG is released from the traps it expands freely and a matter-wave interference pattern is formed in a few milliseconds. The atom cloud released from one lattice site expands, overlaps and interferes with atom clouds from neighboring sites to form a robust interference pattern, consisting of a central peak and two smaller symmetrically spaced peaks moving in opposite directions along the OL axis [1,38,43. Since the lattice transfers momentum to the SFG in units of $2 p_{R}=2 h / \lambda$, the recoil velocity of each the two side peaks is given by $v_{R}=2 p_{R} /(2 m)=2 h /(2 m \lambda)$ 141]. Using $l^{2}=\hbar /(2 m \omega)$, we have $v_{R}=4 \pi l^{2} \omega / \lambda \approx 10 \mathrm{~mm} / \mathrm{s}$, where we used the numerical values $l \approx 1 \mu \mathrm{m}, \omega^{-1}=1.73 \mathrm{~ms}$, and $\lambda \approx 1 / \sqrt{2}$ $\mu \mathrm{m}$.

To demonstrate the loss of phase correlation after displacing the harmonic trap along the OL direction through $100 \mu \mathrm{m}$, in Figs. 4 we exhibit the contour plot of the 1D probability density $P(z, t)$ of the SFG with $n=225$ released after different hold times $T_{h}$ in the harmonic plus OL traps. In Fig. 4 (a) we exhibit the result of simulation for $T_{h}=0$, which has no effect on the phase correlation. Upon release from the traps a robust interference pattern of the central and two side peaks can be seen in this figure. The side peaks move with velocities $10 \mathrm{~mm} / \mathrm{s}$ and in 12 ms they move about $120 \mu \mathrm{m}$ each as can be seen from this figure. The successive figures 4 (b), (c), and (d) show the situation for hold times $10 \mathrm{~ms}, 30 \mathrm{~ms}$, and $50 \mathrm{~ms}$, respectively. The clean interference pattern of a central and two symmetrically moving side peaks is destroyed slowly with the increase of hold time. For an intermediate $T_{h}(=10 \mathrm{~ms}$ and $30 \mathrm{~ms}$ ) there is partial destruction of the interference pattern. For $T_{h}=50 \mathrm{~ms}$, there is no interference pattern and the expanding SFG occupies the full region between the two side peaks. This simulation illustrates that when the harmonic trap is displaced by a large distance, there is no Josephson oscillation.

The breakdown of Josephson oscillation above is due to a classical transition from a phase-correlated superfluid to an "insulator" resulting in a modulational instability as in a BEC [44. Other mechanisms for the loss of phase coherence in a BEC have also been studied (45). The loss of phase coherence considered in all these investigations 44,45 originated from a classical superfluid to insulator transition, different from a quantum transition of a superfluid to a Mott insulator observed in Ref. 38. The classi- cal phase transition involves energy, whereas the quantum phase transition does not involve a supply of energy.

A recent investigation [46] indicates that, for a BEC, the disruption of the large amplitude oscillations is caused by the onset of dynamical instability occurring when the quasi-momentum surpasses a critical value towards the edge of the first Brilluoin zone. In this regard, it would have been interesting to study what is the critical quasimomentum as a function of the nonlinearity of a SFG. In another experimental study on superfluid Fermi gas 47 with an OL moving at a constant speed, the breakdown of superfluidity was measured as function of speed over the entire Bose to BCS crossover. A careful theoretical investigation on these issues, although beyond the scope of present work, are welcome in the future.

\section{Summary and Conclusion}

We performed 3D numerical simulation based on a timedependent mean-field equation to study Josephson oscillation of a BCS superfluid in a combined axially-symmetric harmonic and periodic OL potentials. The OL potential is aligned along the axial direction and is created by a standing-wave laser beam. The Josephson oscillation is initiated by displacing the harmonic potential along the OL axis by a small distance $(\sim 15 \mu \mathrm{m})$. We study the variation of Josephson frequency with the strength of the OL potential and find that the frequency decreases with the increasing OL strength $s$. As $s$ increases from 1 to 10 the frequency of our SFG model is in agreement with an experiemnt on BEC and on ideal Fermi gas. However, for a large displacement $(\sim 100 \mu \mathrm{m})$ of the harmonic potential along the OL axis we demonstrate a breakdown of Josephson oscillation. The SFG then returns very slowly to the new mean position without executing Josephson oscillation. The breakdown of Josephson oscillation also leads to a destruction of phase correlation and consequent loss of superfluidity in the Fermi gas and is demonstrated by allowing the SFG to undergo a free expansion. The absence of a distinct interference pattern upon free expansion signals the loss of phase correlation due to a classical phase transition to an insulating state as opposed to a quantum superfluid to a Mott insulator transition observed in a BEC 38. Similar superfluid to insulator classical phase transition has been observed [1,44] and studied [5, 45] in a BEC. These features of Josephson oscillation could be verified experimentally and will provide a test for the meanfield model for a SFG.

A proper treatment of SFG should be performed using a fully antisymmetrized many-body Slater determinant wave function 20,33 as in the case of atomic and molecular scattering 48 . However, in view of the success of the hydrodynamic model in other contexts [19,28, 29. we do not believe that the present study on Josephson oscillation of a DFG in an OL potential to be so peculiar as to have no general validity. 
I thank Dr. Paulsamy Muruganandam for help in prepar- 21. R. Roth, Phys. Rev. A 66 (2002) 013614; ing the figures. The work was supported in part by the CNPq and FAPESP of Brazil.

\section{References}

1. F. S. Cataliotti et al., Science 293 (2001) 843.

2. S. V. Pereverzev et al., Nature (London) 388 (1997) 449.

3. F. Dalfovo et al., Rev. Mod. Phys. 71 (1999) 463.

F. Dalfovo, S. Giorgini, L. P. Pitaevskii, S. Stringari, Rev. Mod. Phys. 71 (1999) 463.

4. P. Pedri et al., Phys. Rev. Lett 87 (2001) 220401; S. Burger et al., Phys. Rev. Lett 86 (2001) 4447.

5. S. K. Adhikari, Eur. Phys. J. D 25 (2003) 161.

6. S. K. Adhikari, Phys. Rev. A 72 (2005) 013619.

7. M. Krämer, L. Pitaevskii, S. Stringari, Phys. Rev. Lett. 88 (2002) 180404.

8. I. Lesanovsky, W. vonKlitzing, Phys. Rev. Lett. 98 (2007) 050401;

H. W. Yang, W. Zuo, Chinese Phys. Lett. 24 (2007) 620; H. W. Xiong, S. J. Liu, M. S. Zhan, Phys. Rev. A 74 (2006) 033602;

E. Infeld et al., Phys. Rev. E 74 (2006) 026610;

S. Choi, N. P. Bigelow, Phys. Rev. A 72 (2005) 033612;

A. P. Tonel, J. Links, A. Foerster, J. Phys. A 38 (2005) 6879 ;

M. Krämer et al. Eur. Phys. J. D 27 (2003) 247;

E. Sakellari et al., New J. Phys. 6 (2004) 42;

C. Menotti, A. Smerzi, A. Trombettoni, New J. Phys. 5 (2003) 112;

J. Links, H.-Q. Zhou, Lett. Math. Phys. 60 (2002) 275;

S. Zhang, F. Wang, Phys. Lett. A 279 (2001) 231;

L.-B. Fu, J. Liu, S.-G. Chen, Phys. Lett. A 298 (2002) 388 ;

I. Zapata, F. Sols, and A. J. Leggett, Phys. Rev. A 67 (2003) 021603(R);

S. K. Adhikari and L. Salasnich, Phys. Rev. A 75 (2007) 053603.

9. V. M. Galitski, Phys. Rev. A 72 (2005) 013612;

M. R. Bakhtiari, P. Vignolo, M. P. Tosi, Physica E 33 (2006) 223;

Z. Y. Zhang, J. Phys.: Cond. Matter 18 (2006) 181; M. Wouters, J. Tempere, J. T. Devreese, Phys. Rev. A 70 (2004) 013616;

G. Orso, L. P. Pitaevskii, S. Stringari, Phys. Rev. Lett. 93 (2004) 020404;

G. S. Paraoanu, M. Rodriguez, P. Torma, Phys. Rev. A 66 (2002) 041603(R).

10. L. Pezzè et al., Phys. Rev. Lett. 93 (2004) 120401.

11. B. DeMarco, D. S. Jin, Science 285 (1999) 1703.

12. K. M. O'Hara et al., Science 298 (2002) 2179.

13. F. Schreck et al., Phys. Rev. Lett. 87 (2001) 080403;

A. G. Truscott et al., Science 291 (2001) 2570.

14. Z. Hadzibabic et al., Phys. Rev. Lett. 88 (2002) 160401.

15. G. Modugno et al., Science 297 (2002) 2240.

16. G. Roati et al., Phys. Rev. Lett. 89 (2002) 150403.

17. K. E. Strecker et al., Phys. Rev. Lett. 91 (2003) 080406;

Z. Hadzibabic et al., Phys. Rev. Lett. 91 (2003) 160401.

18. P. Capuzzi et al., Phys. Rev. A 69 (2004) 053615;

P. Capuzzi et al., Phys. Rev. A 68 (2003) 033605.

19. S. K. Adhikari, Phys. Rev. A 70 (2004) 043617.

20. K. Mølmer, Phys. Rev. Lett. 80 (1998) 1804.

R. Roth, H. Feldmeier, Phys. Rev. A 65 (2002) 021603(R); T. Miyakawa et al., Phys. Rev. A 64 (2001) 033611;

22. M. Modugno et al., Phys. Rev. A 68, 043626 (2003). M. Modugno, F. Ferlaino, F. Riboli, G. Roati, G. Modugno, and M. Inguscio, Phys. Rev. A 68, 043626 (2003).

23. C. A. Regal, D. S. Jin, Adv. At. Mol. Opt. Phys. 54 (2007) 1 .

24. D. M. Eagles, Phys. Rev. 186 (1969) 456;

A. J. Leggett, J. Phys. (Paris) Colloq. 41 (1980) C7-19; M. Casas et al, Phys. Rev. B 50 (1994) 15945;

S. K. Adhikari et al, Phys. Rev. B 62 (2000) 8671;

S. K. Adhikari et al, Physica C 453 (2007) 37.

25. M. Greiner, C. A. Regal, D. S. Jin, Nature (London) 426 (2003) 537;

C. A. Regal, M. Greiner, D. S. Jin, Phys. Rev. Lett. 92 (2004) 040403;

J. Kinast et al., Phys. Rev. Lett. 92 (2004) 150402.

26. C. Chin et al., Science 305 (2004) 1128;

M. Bartenstein et al., Phys. Rev. Lett. 92 (2004) 203201;

M.W. Zwierlein et al., Phys. Rev. Lett. 92 (2004) 120403;

M.W. Zwierlein et al., Phys. Rev. Lett. 94 (2005) 180401;

27. D. M. Jezek et al., Phys. Rev. A 70 (2004) 043630.

28. S. K. Adhikari, Phys. Rev. A 72 (2005) 053608; L. Salasnich, S. K. Adhikari, F. Toigo, Phys. Rev. A 75 (2007) 023616.

29. S. K. Adhikari, J. Phys. B 38 (2005) 3607.

30. S. K. Adhikari, B. A. Malomed, Europhys. Lett. 79 (2007) 50003.

31. C. A. Stan et al., Phys. Rev. Lett. 93 (2004) 143001; S. Inouye et al., Phys. Rev. Lett. 93 (2004) 183201.

32. M. Salerno, Phys. Rev. A 72 (2005) 063602.

33. T. Karpiuk et al., J. Phys. B 38 (2005) L215; T. Karpiuk et al., J. Phys. B 36 (2003) L69; T. Karpiuk et al., Phys. Rev. A 69 (2004) 043603.

34. A. L. Fetter and J. D. Walecka, Quantum Theory of ManyParticle Systems, (McGraw-Hill, Boston, 1971).

35. S. K. Adhikari, L. Salasnich, Phys. Rev. A 76 (2007) 023612 .

36. K. Huang, C. N. Yang, Phys. Rev. 105 (1957) 767; T. D. Lee, C. N. Yang, Phys. Rev. 105 (1957) 1119; L. Viverit, C. J. Pethick, H. Smith, Phys. Rev. A 61 (2000) 053605 .

37. N. Manini, L. Salasnich, Phys. Rev A 71 (2005) 033625.

38. M. Greiner et al., Nature (London) 415 (2002) 39.

39. S. K. Adhikari, P. Muruganandam, J. Phys. B 35 (2002) 2831

S. K. Adhikari, Phys. Rev. E 62 (2000) 2937.

40. S. K. Adhikari, Phys. Rev. E 65 (2001) 016703.

41. S. K. Adhikari, J. Phys. B 36 (2003) 3951.

42. S. K. Adhikari, P. Muruganandam, Phys. Lett. A 310 (2003) 229.

43. O. Morsch et al., Phys. Rev. Lett. 87 (2001) 140402.

44. F. S. Cataliotti et al. New J. Phys. 5 (2003) 71.

45. A. Smerzi et al., Phys. Rev. Lett., 80 (2002)170402;

S. K. Adhikari, Phys. Lett. A 313 (2003) 211;

S. K. Adhikari, Phys. Lett. A 308 (2003) 302;

S. K. Adhikari, J. Phys. B 36 (2003) 2725.

46. L. De Sarlo et al., Phys. Rev. A 72 (2005) 013603.

47. D. E. Miller et al., Phys. Rev. Lett. 99 (2007) 070402.

48. P. K. Biswas, S. K. Adhikari, J. Phys. B 33 (2000) 1575; P. K. Biswas, S. K. Adhikari, J. Phys. B 31 (1998) L315; S. K. Adhikari, P. K. Biswas, Phys. rev. A 59 (1999) 2058. 